PROCEEDINGS OF THE

AMERICAN MATHEMATICAL SOCIETY

Volume 139, Number 5, May 2011, Pages 1871-1877

S 0002-9939(2010)10618-4

Article electronically published on November 1, 2010

\title{
A FORMULA ON SCATTERING LENGTH OF DUAL MARKOV PROCESSES
}

\author{
PING HE
}

(Communicated by Richard C. Bradley)

\begin{abstract}
A formula on the scattering length for 3-dimensional Brownian motion was conjectured by M. Kac and proved by others later. It was recently proved under the framework of symmetric Markov processes by Takeda. In this paper, we shall prove that this formula holds for Markov processes under weak duality by the machinery developed mainly by Fitzsimmons and Getoor.
\end{abstract}

\section{INTRODUCTION}

For a non-negative smooth function $V \in L^{1}\left(\mathbb{R}^{3}\right)$, the scattering problem is to find the solutions $\Phi_{k}$ of the Schroedinger equation on $\mathbb{R}^{3}$

$$
\frac{1}{2} \Delta \Phi-V \cdot \Phi=-k^{2} \Phi
$$

$(k \in \mathbb{R})$ which have a certain asymptotic behavior at infinity (see [12]). The function

$$
f_{k}\left(e_{x}\right)=-\frac{1}{2 \pi} \int_{\mathbb{R}^{3}} e^{i k \sqrt{2} y \cdot e_{x}} V(y) \Phi_{k}(y) d y
$$

is called the scattering amplitude, where $x \in \mathbb{R}^{3}$ and $e_{x}$ is the unit vector in the direction of $x$. As $k \rightarrow 0$ (low energy limit) the scattering amplitude becomes independent of $e_{x}$,

$$
f_{0}\left(e_{x}\right)=-\frac{1}{2 \pi} \int V(y) \Phi_{0}(y) d y .
$$

The quantity $\Gamma(V)=-f_{0}\left(e_{x}\right)$ is called the scattering length.

In [11, [12, M. Kac and J. Luttinger gave a probabilistic expression in terms of Brownian motion $B=\left(B_{t}, \mathbb{P}^{x}\right)$ on $\mathbb{R}^{3}$,

$$
\Gamma(V)=\lim _{t \rightarrow \infty} \frac{1}{t} \int_{\mathbb{R}^{3}}\left(1-\mathbb{E}^{x}\left(\exp \left\{-\int_{0}^{t} V\left(B_{s}\right) d s\right\}\right)\right) d x .
$$

In their paper, it was shown that if $K$ is a compact subset of $\mathbb{R}^{3}$ with some kind of regularity, $\Gamma\left(\alpha 1_{K}\right)$ converges to the capacity of $K$ as $\alpha \uparrow \infty$. Moreover Kac conjectured in [11 that $\Gamma(\alpha V)$ also converges to the capacity of the support of $V$, ignoring the concrete form of $V$. This conjecture was proved by M. Taylor 18 probabilistically and by H. Tamura [17] analytically.

Received by the editors March 2, 2010 and, in revised form, May 26, 2010.

2010 Mathematics Subject Classification. Primary 60J40; Secondary 60J45.

This research supported in part by the National Natural Science Foundation of China (Grant No. 10771131).

(C)2010 American Mathematical Society 
In order to extend this formula to the more general case, Takahashi [16] discussed this problem in the case of symmetric Markov processes and asserted that the limit of $\Gamma(\alpha V)$ as $\alpha \rightarrow \infty$ exists and depends only on the support of $V$. More recently still for symmetric Markov processes, Takeda [19] solved the problem completely using the time change techniques of Dirichlet forms and proved that Kac's conjecture holds true. To state his result, let $A$ be a positive continuous additive functional of a Hunt process $X$ on the state space $E$ which is symmetric with respect to $m$ and let $F$ be the fine support of $A$. Then

$$
\lim _{\alpha \uparrow \infty} \lim _{t \uparrow \infty} \frac{1}{t} \int_{E}\left(1-\mathbb{E}^{x}\left[e^{-\alpha A_{t}}\right]\right) m(d x)=\operatorname{Cap}(F),
$$

where Cap is the 0-capacity of Dirichlet form associated with $X$.

In this short article, we shall extend this scattering length formula further to the case of right Markov processes under weak duality. However we believe that the condition of weak duality is technical and may be eliminated due to the existence of the moderate Markovian duality to any right Markov process with respect to an excessive measure. For the theory of the moderate Markov process, we refer to [7] and do not go that far here. The paper is organized as follows. In $\S 2$, a few general results on Revuz measures and energy functionals of Markov processes under duality are presented. The definition of scattering length and its existence are given in $\S 3$. Finally the main result is proved in $\S 4$. What makes this paper more interesting is that we have applied almost all notions in probabilistic potential theory, developed mainly by the San Diego probability group led by P.J. Fitzsimmons and R.K. Getoor, which was collected and stated in [6].

\section{The RevuZ Formula Under DUALity}

Let

$$
X=\left(\Omega, \mathscr{F}, \mathscr{F}_{t}, X_{t}, \theta_{t}, \mathbb{P}^{x}\right)
$$

be a right Markov process on a Radon space $(E, \mathscr{E})$ with transition semigroup $\left(p_{t}\right)$, where $\left(\theta_{t}\right)$ is a semigroup of shift operators for $X$. Roughly speaking, $X$ is a strong Markov process with right continuous sample paths. This guarantees that the potential machinery is valid. Let $m$ be an excessive measure for $X$. Assume that $X$ has a weak duality $\widehat{X}$, another right Markov process on $(E, \mathscr{E})$, with respect to $m$, i.e., for any non-negative measurable function $f, g$ on $E$,

$$
\left(p_{t} f, g\right)_{m}=\left(f, \widehat{p}_{t} g\right)_{m}
$$

where $\left(\widehat{p}_{t}\right)$ is the transition semigroup of $\widehat{X}$. We make the hypothesis that $X$ and $\widehat{X}$ are conservative; i.e., they have infinite lifetimes almost surely. For convenience we always put $\mathrm{a}^{\wedge}$ on when talking about corresponding elements of dual process $\widehat{X}$. For general Markov processes and their potential theory, refer to [1], [6] and [14. The key in Takeda [19] is the Revuz formula, i.e., Theorem 5.1.3 in [5]. Hence in this section, we shall prepare some properties on Revuz measures and energy functionals under the current setting, which will be proved here for completeness.

Let $A=\left(A_{t}\right)$ be a positive continuous additive functional (or PCAF simply) of $X$ and let $\mu$ be the Revuz measure of $A$ with respect to $m$. Let $X^{A}$ denote the subprocess of $X$, killed by the multiplicative functional $\left(e^{-A_{t}}\right)$. Assume further that there exists a positive continuous additive functional $\widehat{A}$ of $\widehat{X}$ such that $\widehat{X}^{\widehat{A}}$ and $X^{A}$ are in weak duality. This is equivalent to saying that the Revuz measure 
of $\widehat{A}$ with respect to $m$ for $\widehat{X}$ is equal to $\mu$ also. Actually a weaker form of dual PCAF might be enough, and the existence of such a dual PCAF was discussed in several papers such as [2] and, [20, §I.6]. A main tool used in the duality situation is the energy functional of a Markov process, which is introduced by P.A. Meyer and discussed carefully in [6]. The energy functional is defined on a pair of an excessive measure and an excessive function, and it may play the role of the Dirichlet form in the symmetric case. Let $L^{X}$ and $L^{\widehat{X}}$ denote the energy functionals of $X$ and $\widehat{X}$, respectively. The following two lemmas were stated and proved in [10].

Lemma 2.1. If $h$ and $\widehat{h}$ are excessive for $X$ and $\widehat{X}$ respectively, then

$$
L^{X}(\widehat{h} \cdot m, h)=L^{\widehat{X}}(h \cdot m, \widehat{h}) .
$$

The next result is the generalized form of the Revuz formula and is also called a switching identity in [10], since it indicates how additive functionals switch. The original Revuz formula is

$$
\int_{E} g(x) m(d x) \mathbb{E}^{x}\left[A_{t}\right]=\int_{E} \mu(d x) \widehat{\mathbb{E}}^{x} \int_{0}^{t} g\left(\widehat{X}_{s}\right) d s, \text { for all } t>0
$$

or equivalently using Laplace transform, for any $\alpha>0$,

$$
\int_{E} g(x) m(d x) \mathbb{E}^{x} \int_{0}^{\infty} e^{-\alpha t} d A_{t}=\int_{E} \mu(d x) \widehat{\mathbb{E}}^{x} \int_{0}^{\infty} e^{-\alpha t} g\left(\widehat{X}_{t}\right) d t .
$$

The formula in the next lemma is clearly more powerful.

Lemma 2.2. If $L$ and $\widehat{K}$ are positive continuous additive functional of $X$ and $\widehat{X}$, respectively, we have the following switching identity, for any non-negative measurable functions $f, g$ on $E$ :

$$
\begin{aligned}
\int_{E} g(x) \mathbb{E}^{x}\left[\int_{0}^{t} e^{-A_{s}} f\left(X_{s}\right) d L_{s}\right] \mu_{\widehat{K}}(d x) \\
=\int_{E} \widehat{\mathbb{E}}^{x}\left[\int_{0}^{t} e^{-\widehat{A}_{s}} g\left(\widehat{X}_{s}\right) d \widehat{K}_{s}\right] f(x) \mu_{L}(d x),
\end{aligned}
$$

where $\mu_{L}$ and $\mu_{\widehat{K}}$ are the Revuz measures of $L$ and $\widehat{K}$, respectively, with respect to $m$ under their corresponding processes.

We give a brief proof here which is a little different from the one in [10. Since $U_{L}^{\alpha} f=\mathbb{E} \cdot \int_{0}^{\infty} e^{-\alpha t} f\left(X_{t}\right) d L_{t}$ and $\widehat{U}_{\widehat{K}}^{\alpha} g=\mathbb{E} \cdot \int_{0}^{\infty} e^{-\alpha t} g\left(\widehat{X}_{t}\right) d \widehat{K}_{t}$ are $\alpha$-excessive and coexcessive respectively, it follows from (2.1), (8.7) in [8] and Meyer's representation theorem 13 that

$$
\begin{aligned}
\mu_{L}\left(f \cdot \widehat{U}_{\widehat{K}}^{\alpha} g\right) & =L^{X^{\alpha}}\left(\widehat{U}_{\widehat{K}}^{\alpha} g \cdot m, U_{L}^{\alpha} f\right) \\
& =L^{\widehat{X}^{\alpha}}\left(U_{L}^{\alpha} f \cdot m, \widehat{U}_{\widehat{K}}^{\alpha} g\right)=\mu_{\widehat{K}}\left(g \cdot U_{L}^{\alpha} f\right) .
\end{aligned}
$$

This is a special case of (2.4) when $A=0$. Note that by Theorem 2.22 in (4), the Revuz measure of a positive continuous additive functional computed against a subprocess is equal to the one computed against $X$ as long as the subprocess has the same state space as $X$ and hence is independent of $\alpha$. Considering the formula (2.5) for dual subprocesses $X^{A}$ and $\widehat{X}^{\widehat{A}}$ and applying Theorem 2.22 in [4] again, the desired formula (2.4) follows. 


\section{The EXISTEnCE OF SCATTERING LENGTH}

We shall start from the probabilistic expression of scattering length in [12], which makes sense for general Markov processes. Let $A$ be a positive continuous additive functional of $X$ as in $\S 2$ with Revuz measure $\mu$. Define

$$
\Gamma(A)=\lim _{t \uparrow+\infty} \frac{1}{t} \int_{E}\left(1-\mathbb{E}^{x}\left[e^{-A_{t}}\right]\right) m(d x),
$$

which we call the scattering length of $A$ directly. For the first step, we shall prove that the limit above exists and we shall give another expression for $\Gamma(A)$.

Lemma 3.1. If $\mu(E)<\infty$, then

$$
\Gamma(A)=\int_{E} \widehat{\mathbb{E}}^{x}\left[e^{-\widehat{A}_{\infty}}\right] \mu(d x) .
$$

Proof. Clearly

$$
1-\mathbb{E}^{x}\left[e^{-A_{t}}\right]=\mathbb{E}^{x} \int_{0}^{t} e^{-A_{s}} d A_{s} .
$$

Taking $d \widehat{K}_{s}=d s, d L_{s}=d A_{s}$ and $f=g=1$ in the Revuz formula (2.4), we have

$$
\int_{E} \mathbb{E}^{x}\left[\int_{0}^{t} e^{-A_{s}} d A_{s}\right] m(d x)=\int_{E} \mu(d x) \widehat{\mathbb{E}}^{x}\left[\int_{0}^{t} e^{-\widehat{A}_{s}} d s\right] .
$$

Since $t \mapsto \widehat{A}_{t}$ is increasing to $\widehat{A}_{\infty}$, it follows from the dominated convergence theorem that

$$
\begin{aligned}
\Gamma(A) & =\lim _{t \uparrow \infty} \frac{1}{t} \int_{E} \mu(d x) \widehat{\mathbb{E}}^{x} \int_{0}^{t} e^{-\widehat{A}_{s}} d s \\
& =\int_{E} \widehat{\mathbb{E}}^{x}\left[e^{-\widehat{A}_{\infty}}\right] \mu(d x) .
\end{aligned}
$$

That completes the proof.

The finiteness of $\mu$ in the result is natural. Actually in the simple case when $A_{t}=\int_{0}^{t} V\left(X_{s}\right) d s$ with $V \geq 0, \mu(d x)=V(x) m(d x)$, and hence the condition $\mu(E)<\infty$ amounts to saying that $V \in L^{1}(E ; m)$, which appeared originally in [11.

\section{MAin RESUlT}

Let us now consider the limit of $\Gamma(\alpha A)$ when $\alpha \uparrow \infty$ and prove the main result in this paper. We also need to use the time change approach as in [19. It is known that a PCAF induces a time change. More precisely, let $\left(\tau_{t}\right)$ be the right continuous inverse of $\left(A_{t}\right)$, namely

$$
\tau_{t}=\inf \left\{s>0: A_{s}>t\right\}, t \geq 0 .
$$

Let $Y_{t}=X_{\tau_{t}}, t \geq 0$, and let $F$ be the fine support of $A$, i.e.,

$$
F=\left\{x \in E: \mathbb{P}^{x}\left(\tau_{0}=0\right)=1\right\},
$$

which is nearly Borel and finely perfect. Intuitively $A$ moves immediately when $X$ starts at a point of $F$. Actually $\mu$ is supported on $F$. Then $Y=\left(Y_{t}\right)$ is the time change process of $X$ induced by $A$ and is also a right Markov process on $F$; see 14. Finally $\zeta_{Y}=A_{\infty}$ is the lifetime of $Y$. Similarly $\widehat{Y}=\left(Y_{t}\right)$ is the time change process of $\widehat{X}$ induced by $\widehat{A}$. The following duality is actually stated in Lemma 5.2 
of [3. However the proof there is a little unnecessarily complicated, and here we shall show that it is a simple and direct consequence of (2.4).

Lemma 4.1. The time change processes $Y$ and $\widehat{Y}$ are dual with respect to $\mu$.

Proof. Let $\left(G^{\alpha}\right)$ and $\left(\widehat{G}^{\alpha}\right)$ be the resolvents of $Y$ and $\widehat{Y}$, i.e.,

$$
\begin{aligned}
& G^{\alpha} f(x)=\mathbb{E}^{x} \int_{0}^{\infty} e^{-\alpha t} f\left(Y_{t}\right) d t=\mathbb{E}^{x} \int_{0}^{\infty} e^{-\alpha A_{t}} f\left(X_{t}\right) d A_{t}, \\
& \widehat{G}^{\alpha} f(x)=\widehat{\mathbb{E}}^{x} \int_{0}^{\infty} e^{-\alpha \widehat{A}_{t}} f\left(\widehat{X}_{t}\right) d \widehat{A}_{t}
\end{aligned}
$$

for any non-negative measurable function $f$ on $E$. Taking $t=\infty, L=A$ and $\widehat{K}=\widehat{A}$ in (2.4) and observing the fact that $\mu$ is the Revuz measure of both $A$ and $\widehat{A}$, we have

$$
\left(g, G^{\alpha} f\right)_{\mu}=\left(\widehat{G}^{\alpha} g, f\right)_{\mu}, \alpha>0,
$$

which implies the duality of $Y$ and $\widehat{Y}$ with respect to measure $\mu$.

To state our main result, we need to introduce the capacity. For any $B \in \mathscr{E}$, nearly Borel, $T_{B}$ denotes the hitting time of $B$ and

$$
P_{B}(x, d y)=\mathbb{P}^{x}\left(X_{T_{B}} \in d y, T_{B}<\infty\right),
$$

which is the hitting distribution of $X$ on $B$. Let $L$ be the energy functional of $X$ and then the capacity of $B$ with respect to $m$ is defined by

$$
\operatorname{Cap}(B)=L\left(m, P_{B} 1\right) \text {. }
$$

For these elements of potential theory, refer to [6]. If necessary, we put a superscript to indicate the connection of these elements to specific processes; for instance, $L^{Y}$ is the energy functional of $Y$. The following properties of energy functional may refer to 4] or Theorem 2.1 of [9].

Lemma 4.2. If $Y$ is the time change process of $X$ induced by $A$ with fine support $F$ and if $h$ is an excessive function for $Y$, then

$$
L^{Y}(\mu, h)=L^{X}\left(m, P_{F} h\right) .
$$

We are now in a position to present our main result.

Theorem 4.1. Let $A$ be a positive continuous additive functional of $X$ with fine support $F$. If $\mu(E)<\infty$, then

$$
\lim _{\alpha \uparrow \infty} \Gamma(\alpha A)=\operatorname{Cap}(F) .
$$

Proof. Since $\widehat{A}_{\infty}$ is the lifetime of $\widehat{Y}$,

$$
\begin{aligned}
\widehat{\mathbb{E}}^{x}\left[e^{-\alpha \widehat{A}_{\infty}}\right] & =1-\alpha \widehat{\mathbb{E}}^{x}\left[\int_{0}^{\widehat{\zeta}_{Y}} e^{-\alpha t} d t\right] \\
& =1-\alpha \widehat{\mathbb{E}}^{x}\left[\int_{0}^{\infty} e^{-\alpha t} 1_{F}\left(\widehat{Y}_{t}\right) d t\right]=1-\alpha \widehat{G}^{\alpha} 1_{F}
\end{aligned}
$$

Furthermore $\mu$ is supported on $F$ and then

$$
\begin{aligned}
\lim _{\alpha \rightarrow \infty} \Gamma(\alpha A) & =\lim _{\alpha \rightarrow \infty} \alpha\left\langle\mu, 1_{F}-\alpha \widehat{G}^{\alpha} 1_{F}\right\rangle \\
& =L^{\widehat{Y}}\left(\mu, 1_{F}\right),
\end{aligned}
$$


where $L^{\widehat{Y}}$ is the energy functional of $\widehat{Y}$. The second equality above follows formally from (3.6)(i) in [6], but not really, because it is not evident that the conditions for this to is true are satisfied. Suppose that (4.3) is true. Lemma 4.1 tells us that $Y$ and $\widehat{Y}$ are dual under $\mu$, and hence by Lemma 2.1.

$$
L^{\widehat{Y}}\left(\mu, 1_{F}\right)=L^{Y}\left(\mu, 1_{F}\right) .
$$

Then by the assertion in Lemma 4.2, it follows that

$$
L^{Y}\left(\mu, 1_{F}\right)=L\left(m, P_{F} 1\right)=\operatorname{Cap}(F) .
$$

To complete the proof we need to justify (4.3). The following approach is provided by the referee. Recall that $m$ admits a unique decomposition as $m=m_{c}+m_{d}$, the sum of the conservative part and dissipative part (see page 8 of $[$ ). Since the energy functional and Revuz measure are linear for $m$, it suffices to verify (4.3) for $m$ to be conservative and dissipative.

If $m$ is conservative for $X$, then $\mu$ is conservative for $\widehat{Y}$ by (4.15)(i) in [4. In particular, $\widehat{Y}$ has infinite lifetime for $\mu$-a.e. starting point by (2.9) in 6 . Hence both sides of (4.3) are zero. On the other hand, if $m$ is dissipative, there exists a sequence $\left\{\mu_{n}\right\}$ of finite measures, each equivalent to $m$, such that $\mu_{n} U \uparrow m$. We then have

$$
\infty>\mu(E)=L\left(m, U_{A} 1\right)=\lim _{n} L\left(\mu_{n} U, U_{A} 1\right)=\lim _{n} \mu_{n}\left(U_{A} 1\right) .
$$

It follows that $\mathbb{P}^{x}\left(A_{\infty}\right)=U_{A} 1(x)<\infty$ for $m$-a.e. $x \in E$ and also for $m$-q.e. $x \in E$ since $U_{A} 1$ is excessive. This implies that $\mathbb{P}^{x}\left(A_{\infty}<\infty\right)=1$ for $m$-q.e. $x \in E$. Consequently $Y$ has finite lifetime for $\mu$-q.e. starting point and $\mu$ is a purely excessive measure for $\widehat{Y}$. Hence applying (3.6)(i) in [6], (4.3) holds.

\section{ACKNOWLEDGEMENTS}

The author would like to thank Professor Jiangang Ying for his great help during the preparation of this paper and especially to thank the anonymous referee for valuable suggestions. Actually the current proof of (4.3) was provided by the referee.

\section{REFERENCES}

1. R.M. Blumenthal, R.K. Getoor, Markov Processes and Potential Theory, Academic Press, 1968. MR0264757 (41:9348)

2. R.M. Blumenthal, R.K. Getoor, Additive functionals of Markov processes in duality. Trans. Amer. Math. Soc. 112 (1964), 131-163. MR0160269 (28:3483)

3. Z. Chen, M. Fukushima, J. Ying, Entrance law, exit system and Lévy system of time change processes, Illinois J. Math. 50, 2 (2006), 269-312. MR2247830 (2007k:60242)

4. P.J. Fitzsimmons and R.K. Getoor, Revuz measures and time changes, Math. Zeit. 199 (1988), 233-256. MR958650 (89h:60124)

5. M. Fukushima, Y. Oshima and M. Takeda, Dirichlet Forms and Symmetric Markov Processes, De Gruyter, 1994. MR.1303354 (96f:60126)

6. R.K. Getoor, Excessive measures, Birkhäuser, 1990. MR.1093669 (92i:60135)

7. R.K. Getoor, Duality Theory for Markov Processes, Part I, preprint, 2010.

8. R.K. Getoor, M.J. Sharpe, Naturality, standardness, and weak duality for Markov processes, Z. W. Verw. Geb. 67 (1984), 1-62. MR756804 (86f:60093)

9. P. He, J. Ying, Revuz measure under time change, Sci. China. Ser. A 51 (2008), 321-328. MR 2395426 (2009e:60178)

10. M.W. Jin, J. Ying, Additive functionals and perturbation of semigroup. Chinese Ann. Math. Ser. B 22 (2001), no. 4, 503-512. MR1870075 (2003f:60137) 
11. M. Kac, Probabilistic methods in some problems of scattering theory, Rocky Mountain J. Math. 4 (1974), 511-537. MR0510113 (58:23170)

12. M. Kac and J.-M. Luttinger, Scattering length and capacity, Ann. Inst. Fourier (Grenoble) 25 (1975), 317-321. MR0402079 (53:5902)

13. P.A. Meyer, Note sur l'interpretation des mesures d'équilibre. Sém. de Prob. VII. Lecture Notes in Math. 321, pp. 210-216, Springer, Berlin, 1973. MR0373030 (51:9232)

14. M. Sharpe, General theory of Markov processes, Pure and Applied Mathematics, 133, Academic Press, 1988. MR958914 (89m:60169)

15. D.W. Stroock, The Kac approach to potential theory. I, J. Math. Mech. 16 (1967), 829-852. MR0208690 (34:8499)

16. Y. Takahashi, An integral representation on the path space for scattering length, Osaka J. Math. 7 (1990), 373-379. MR1066632 (91j:35083)

17. H. Tamura, Semi-classical limit of scattering length, Lett. Math. Phys. 24 (1992), 205-209. MR.1166749 (93m:81030)

18. M.E. Taylor, Scattering length and perturbations of $-\Delta$ by positive potentials, J. Math. Anal. Appl. 53 (1976), 291-312. MR0477504 (57:17028)

19. M. Takeda, A formula on scattering length of positive smooth measures, Proc. Amer. Math. Soc. 138 (2010), 1491-1494. MR 2578543

20. J. Ying, Bivariate Revuz measure and the Feynman-Kac formula, Ann. Inst. Henri Poincaré 32, 2 (1996), 251-287. MR 1386221 (97j:60139)

Department of Applied Mathematics, Shanghai University of Finance and Economics, Shanghai, 200433, People's Republic of China

E-mail address: pinghe@mail.shufe.edu.cn 